\title{
A preliminary analysis of learning awareness in FLOSS projects
}

\author{
Sara Fernandes ${ }^{1,2}$, Antonio Cerone ${ }^{1}$, Luis Soares Barbosa ${ }^{2}$ \\ ${ }^{1}$ United Nations University - International Institute for Software Technology, Macao \\ SAR \\ ${ }^{2}$ HASLab / INESC TEC, University of Minho \\ ${ }^{1}$ \{sara.fernandes, antonio\}@iist.unu.edu, ${ }^{2}$ lsb@di.uminho,pt
}

\begin{abstract}
It can be argued that participating in free/libre open source software (FLOSS) projects can have a positive effect in the contributor's learning process. The need to interact with other contributors, to read other people's code, write documentation, or use different tools, can motivate and implicitly foster learning. In order to validate this statement we design an appropriate questionnaire asking FLOSS contributors about their experience in FLOSS projects. In this paper, we illustrate how this questionnaire was designed and what we expect to learn from the answers. We conclude the paper with a preview of the results from three cases studies.
\end{abstract}

Keywords: FLOSS participants, FLOSS Community, Communities of practice, Learning awareness

\section{Introduction}

Free/Libre Open Source Software (FLOSS) communities consist of heterogeneous groups of independent volunteers, who interact even if driven by different motivations [3]. FLOSS provides an example of peer-production [9], which is based on collaborative, social modes of interaction and knowledge exchange [4].

The above characteristics raise a number of questions relevant to Education. In particular, one may wonder in what sense contributors to FLOSS projects learn through collaboration and collaborate while learning [3].

Do FLOSS contributors recognize their FLOSS communities as possible learning environments? Do the activities performed in a FLOSS community enable knowledge sharing and collaborative learning experiences? Such are certainly pertinent questions. In fact, FLOSS communities collaborate in a wide range of activities, which are by no means restricted to software development. Such activities include exchange of information, reciprocal support and technical assistance, planning, code review, testing, and use and sometimes even development of several kinds of tools [10]. Therefore, it makes sense to understand how people learn and produce knowledge, and how communities collaborate and work to solve problems while performing these activities. 
FLOSS can be regarded as an instantiation of the Common-based Peer Production (CBPP) model. CBPP [8] refers to the collaborative efforts based on sharing information and outcomes of a large number of people working incrementally on a problem [2]. It constitutes a new model of economic production based on a theory that, in addition to market, signals or managerial commands, individuals can organize their productive behavior based collective action. The latter occurs when large numbers of people work independently on a single project "following a diverse cluster of motivation drives and social signals" often without traditional hierarchical organization or financial compensation [6]. More specifically, CBPP can be defined as any coordinated mainly "internet-based efforts whereby volunteers contribute project components, and there exists some process to combine them to produce a unified intellectual work" [7].

Yochai Benkler, who first introduced the CBPP model, considered FLOSS "as an emerging third model of production which maximizes human creativity". Dillon identifies four key characteristics of CBPP that underlie the FLOSS approach to software production [3]:

- Motivation where individuals are not driven by money to work on a project;

- Chunking where many individuals work incrementally and asynchronously on a problem;

- Multi-disciplinary where peer-production projects include a large number of contributors, complementing expertise and solving problems;

- Integration of successful peer production products.

Hence, FLOSS can be considered as the prime example of CBPP [9].

In this context, this paper presents three preliminary case studies, which indicate the potential positive impact of membership to a FLOSS community on the contributor's learning processes.

The FLOSS community itself provides a valuable, though partial, source of information. This paper focuses on the FLOSS community and its contributors. As a way to analyze whether FLOSS has a positive effect on contributors' learning we decided to run an online questionnaire directed to a few FLOSS contributors. The results, although with no statistical relevance, may pave the way to further, broader investigations.

The rest of the paper is structured as follows. Section 2 introduces the motivations and scope of the questionnaire. Section 3 presents some of its results. Finally, Section 4 concludes and points to the envisaged future work.

\section{Approach}

Our hypothesis is that long-term participation in FLOSS projects can have a positive effect in the contributor's learning process [3]. Thus, our main goal with this questionnaire is to understand how contributors learn and whether they recognize the learning process they experience. Hence, the questionnaire is focused on gathering evidence relative to questions like the contributors' ability to interact with other contributors, his/her documentation of his/her own achievements or doubts, his/her 
level of commitment in the FLOSS project, and, ultimately, contributors' awareness of learning mechanisms within their contribution to the project.

We divided the questionnaire into three sets of questions.

The first set of questions is titled "About you as a FLOSS contributor" and concerns personal information (see Table 1, items 1-6). Contributors are not only requested to present themselves (name, academic background, etc.), but also to describe a list of FLOSS projects to which they have contributed (item 7 in Table 1). Examples of questions are: "Age", "Country", "Education level", and "List all FLOSS projects that you were involved in". Out of this first set of questions we expect to characterize types of contributors in terms of their background and level of involvement in FLOSS projects.

Table 1. Questionnaire - Respondents' demographic data

\begin{tabular}{l|l|l}
\hline Section A - About you as a FLOSS contributor & 7. FLOSS Communities/ Projects \\
\hline 1. Age & 4. Language & \\
2. Gender & 5. Education & \\
3. Country & 6. Career & \\
\hline
\end{tabular}

The second set of questions is titled "Your involvement as a FLOSS contributor" and is related to a specific project chosen by the contributor. Example questions are shown in Table 2. In the first set of questions, the contributor describes the project and its community (1-9). The second set aims at assessing his/her type of commitment with the community. Questions include: the date in which he/she joined the community (10), the motivation to join (11), initial and current role (12-13), number of daily hours dedicated to the project (14), frequency for committing (15) or releasing (16) contributions, and perception of the usefulness of the documentation produced for himself (17) and other members of the community (18). The third group of questions assesses the interactions between community members including: type of relationships (19); frequency of communications (20) and channels used (21); existence, type and frequency of meetings with other members (22-23); existence and type of conflicts with other communities (24-25); existence of conflicts between members of the community (26) and the approach for managing them (27); events organized by the community (28-29). Finally, the last question assesses the respondent's perception on the quality of results (30).

Table 2. Questionnaire - Respondent's contribution to FLOSS

Section B - Your involvement as a FLOSS contributor

\begin{tabular}{l|l|l}
\hline 1. Project Name & 11. Personal Motivation & 21. Communication channel \\
2. Project Description & 12. Initial role & 22. Meetings with members \\
3. Project Field/Area & 13. Current role & 23. Meetings - types, frequency \\
4. Project initiation date & 14. Hours per day & 24. Conflicts w/ other communities \\
5. Community name & 15. Frequency of commits & 25. Kind of conflicts \\
6. Community group & 16. Frequency of releases & 26. Conflicts with members \\
7. Community size & 17. Documentation - personal use & 27. Conflict management \\
8. Active community size & 18. Documentation - community use & 28. Events organization \\
9. Community structure & 19. Relationships with members & 29. Types of organized events \\
10.Contribution initiation date & 20. Communication frequency & 30. Perception on quality of results \\
\hline
\end{tabular}

Questions included in this section are: 1) open, i.e. "What is the project name?"; 2) closed, i.e. "How many members does the community have" - options include: less 
than 5, between 5 and 10, and more than 10; and 3) open-ended; i.e. "What sort of relationships do you have with the other community members" - options include: we are all friends; we just work together; I don't know community members; others, please specify.

The third set of questions is titled "FLOSS communities as possible learning contexts". It inquires on FLOSS communities as possible learning contexts, as well as potential educational frameworks. The nature of the questions is presented in Table 3 . They include questions such as: "Did the fact of being in a FLOSS community provide any relevant learning opportunity for you?" (1); "What did you learn while engaged in a FLOSS project and its community?" (2); or "Did your involvement as a contributor to this project changed the way you assess your own formal education?" (13). In general, the objective was to invite FLOSS contributors to assess their own experience as learners while members of a community and along their whole involvement in the project. The nature of questions is presented in Table 3.

Table 3. Questionnaire - FLOSS as a learning context

\begin{tabular}{l|l|l}
\hline \multicolumn{2}{l}{ Section C - FLOSS communities as possible learning contexts } \\
\hline 1. Learning opportunity & 6. Learning process & 11. Acquired competencies \\
2. Type of learning & 7. Contribution of role & 12. Contribution to educational aim \\
3. Contribution of background & 8. Knowledge sharing & 13. Contribution to formal education \\
4. Learning agents & 9. Knowledge sharing tools & 14. Complementarity to formal \\
5. Relevance of learning activities & 10.Knowledge sharing areas & education \\
\hline
\end{tabular}

\section{Partial Results - Case Studies}

We tested the questionnaire on a limited number of persons. The questionnaire was sent to 15 active FLOSS contributors; 5 responses were received. We consider here three of these individuals and present them as case studies.

Case 1 - The first case study concerns A., a $\mathrm{PhD}$ student, male, who has been contributing to several FLOSS projects, including Perl, Parrot and Debian.

For analysis he selected the Parrot project. Parrot is a register-based process virtual machine designed to run dynamic languages. The project started in 2006 and the community is called Parrot users. It has more than 10 active members.

A. became a member of this community as a tester, but currently he is a developer. The means this community uses to communicate include wikis, forums and chats. Moreover, the community has vis-à-vis meetings.

A. Agrees that a FLOSS project and its community can be regarded as a learning environment, where he is able to develop skills as a tester, programmer, code reviewer and/or analyst. He is aware of the fact that his background highly contributes to his success within this specific FLOSS project. He also reported in the questionnaire that FLOSS project communities had a relevant role in his learning experience. For him the community has "a lot of knowledge that can be externalized and shared". He also states that the involvement in a FLOSS project can be regarded as a possible alternative and/or a complement to formal education. Alternative to formal education because the contributor has the opportunity to work with up-to-date 
programming languages, applications and technologies, and gets support in his FLOSS community for mastering them. Complementary to formal education because it can be regarded as a practical assessment of the learning process. The feedback given by A. is positive and, for him, a FLOSS project can have a positive effect in contributor's learning process.

Case 2 - B. is a male who currently works as a software developer. He contributed to several FLOSS projects, such as, Gwibber, Bigodejs, Plone and Django. He holds an undergraduate degree in Mathematics and Computer Sciences.

He selected the Plone project for this questionnaire. Plone is a Web CMS based on the Zope application server. This project was created in 2001 and involves currently more than 10 active members. B. became a member of this community as an observer, driven by the novelty and the challenge. Currently he is a developer. The community uses mailing lists as the main communication means.

For B. a FLOSS community provides a relevant learning opportunity. He recognizes he has improved skills such as testing, programming and performing code analysis while contributing to the community. He also perceives his interactions with the community as a good way to share knowledge. He recognizes that his involvement in FLOSS projects enabled him to understand how FLOSS communities work and develop software and, consequently, changed the way he thinks about software engineering education in general. It also contributed to change the way he assesses his own formal education.

However, he does not regard his involvement in FLOSS projects as an alternative to formal education but as a complement that provides him with an environment in which usability and collaboration skills can be improved. The feedback given is, in general, positive and, for B., a FLOSS project can have a positive effect in contributor's learning process if suitably combined with formal education.

Case 3 - C. is a male who holds a $\mathrm{PhD}$ in computer science. He is currently a researcher in Natural Language Processing. He worked for several FLOSS projects, such as, Perl Dancer, Perl Lingua:: Jspell, Perl Lingua:: NATools and Perl Lingua::FreeLing3.

In the second set of questions he selected the Perl Dancer project. Perl Dancer is a web framework based on Sinatra. This project community is called Perl Community. Although this project exists since 2008 it has currently less then 5 active members. C. became a member of this community as an observer, curious of the topic. Currently he is a developer and considers producing documentation as a very important activity. The communication means of this community include chats, mailing lists and wikis. The community members physically meet once a year and have regular online meetings.

C. recognizes that taking part in a FLOSS community provided him with an important learning opportunity as a tester, programmer, code reviewer and analyst. He shares his knowledge of programming and writing documentation with the community members through wikis, mailing lists and chat.

C. does not feel that the Perl Dancer project contributed to change the way he assesses formal education. He still thinks universities should teach how to think independently about methods, tools and technologies. However, he also believes that FLOSS communities can be regarded as educational communities, since they provide a platform for learning technologies as well as technical and social skills. In his 
opinion a FLOSS project cannot be considered as an alternative to formal education but represents a complement to it.

We can observe that in all three case studies the subject (1) regards his participation in the FLOSS project as a learning experience; (2) recognizes to have started participation in the community either as a tester or observer rather than directly as a developer; (3) is currently able to perform a much larger variety of tasks than before: testing, programming, code review, code analysis or writing documentation.

These observations suggest that individuals' participation in a FLOSS community evolves through time. The initial stage of such evolution is characterized by activities that aim to observe community processes rather than producing code. Therefore, the evolution toward more mature stages of contribution, as described by A., B. and C., can be seen as potential learning process.

Another interesting observation is, in case 3, that $\mathrm{C}$., who belongs to the smallest community, perceives that the quality of the product developed in his project is just average. A. and B., in cases 1 and 2, respectively, who belong to communities with more than 10 members, claim instead the very high quality of the products developed in their project. This seems to confirm what Raymond wrote in the essay The Bazaar and the Cathedral: "given enough eyeballs, all bugs are shallow" [5].

\section{Conclusion and Future Work}

The goal the questionnaire briefly described in this paper was to understand whether and to which extent participation in FLOSS projects may effectively contribute to the individual's learning process.

Although the questionnaire is still in a testing phase, we can observe that in the three cases discussed above a consensus emerged on the fact that FLOSS communities provide experiences that can be regarded as valuable complements to formal education. Furthermore, all of them express the belief that participation in FLOSS projects fosters the development of competences in important areas, such as usability, that are seldom addressed by formal education.

The three case studies figure out participation in a FLOSS community as a good way to share knowledge and develop skills. All three feel that knowledge sharing occurs naturally and easily within FLOSS communities. We conjecture that, to a great extent, this happens because of contributors' freedom in joining projects, a choice which is usually supported on strong intrinsic and extrinsic motivations.

Since the questionnaire is still in its testing phase, such statements rely on weak results and need further validation. Therefore, as part of our future work, we intend to improve the questionnaire and apply it to a significant sample of FLOSS contributors. Also, we intend to distinguish between formal, informal, and non-formal aspects of learning, as in recent approaches to life-long learning contexts. Moreover, since this is an online questionnaire, we anticipate the need to run personal interviews to selected targets to tune results. 
Acknowledgments. We would like to thank Pantelis Papadopoulos for helpful discussions. This work is funded partly by UNU-IIST and Macau Foundation in the context of the PPAeL project, as well as by ERDF - European Regional Development Fund through the COMPETE Programme (operational programme for competitiveness) and by National Funds through the FCT (Portuguese Foundation for Science and Technology) within project FCOMP-01-0124-FEDER-007254.

\section{References}

[1] Kumar, Ranjit. (2005). Research Methodology-A Step-by-Step Guide for Beginners (2 ${ }^{\text {nd }}$ ed.). Singapore. Pearson Education.

[2] Benkler, Yochai. (2006). The Wealth of Networks: How Social Production Transforms Markets and Freedom. New Haven, Conn: Yale University Press.

[3] Cerone, A. \& Sowe, S. K. (2010). Using Free/Libre open Source Software Projects as Learning Tools. OpenCert 2010, Vol. 33 of ECEASST.

[4] Dillon, Teresa. (2006), The potential of open source approaches for education. Retrieved from http://archive.futurelab.org.uk/resources/publications-reportsarticles/opening-education-reports/Opening-Education-Report200 in May 2012.

[5] Eric S. Raymond. (1999). The Cathedral and the Bazaar (1st ed.). Tim O'Reilly (Ed.). O'Reilly \& Associates, Inc., Sebastopol, CA, USA.

[6] Krowne, Aaron. (2005). The FUD-based Encyclopedia: Dismantling fear, uncertainty, and doubt, aimed at Wikipedia and other free knowledge resources. Free Software Magazine, N. 2.

[7] Benkler, id. (2005). Common Wisdom: Peer Production of Educational Materials. COSL Press.

[8] Krowne, Aaron. (2005). "The FUD based encyclopedia: Dismantling the Fear, Uncertainty and Doubt aimed at Wikipedia and other free knowledge sources". Free Software Magazine.

[9] Magrassi, Paolo. (2010). Free and Open-Source Software is not an Emerging Property but Rather the Result of Studied Design. ICICKM10.

[10] Conole, Gráinne, Littlejohn, Allison, Falconer, Isabel and Jeffery, Ann. (2005). Pedagogical review of learning activities and use cases. LADIE project report (August).

[11] Crowston, K., Wei, K., Howison, J., and Wiggins, A. Free/Libre Open Source Software Development: What We Know and What We Do Not Know. Retrieved from

http://crowston.syr.edu/system/files/CrowstonFLOSSReviewPaperPreprint.pdf in July 2012. 